\title{
Protection of Caridea Against White Spot Syndrome Virus $^{\dagger}$
}

\author{
Masilamani Selvam M. ${ }^{1}$, Sherin Sangeetha S. ${ }^{1}$, Ubagara Blesscy A. ${ }^{1}$, Sravya P. ${ }^{1, *}$ \\ 1 Department of Biotechnology, Sathyabama Institute of Science and Technology, Jeppiaar Nagar, Rajiv Gandhi Salai, \\ Chennai - 600 119, Tamil Nadu, India \\ * Correspondence: sravyashanu2000@gmail.com; \\ $\dagger$ Presented at International e-Conference on Bioengineering for Health and Environment (ICBHE 2020) \\ Received: 5.07.2020; Revised: 10.07.2020; Accepted: 12.07.2020; Published: 15.07.2020
}

\begin{abstract}
White spot syndrome virus (WSSV) belongs to a new virus family, Nimaviridae, genus Whispovirus and contains a large circular double-stranded DNA genome of 292,967 bp. WSSV virions are ellipsoid to bacilliform, enveloped particles with a distinctive tail-like appendage at one end. They can be found throughout the body of infected shrimp. The virions contain one nucleocapsid with a typical striated appearance and 5 major and at least 13 minor proteins. WSSV, which was first discovered in Southeast Asia around 1992, is currently the most serious viral pathogen of shrimp worldwide. It causes up to $100 \%$ mortality within 7 to 10 days in commercial shrimp farms, resulting in large economic losses amounting to billions of US dollars across different countries to the shrimp farming industry. In a natural situation, shrimp become infected through both oral and water-borne routes, and the gills are thought to be a major point of viral entry. Considering the global economic and sociological importance of shrimp farming and its continued high growth, the development of novel control measures becomes necessary against the outbreak of WSSV. A number of strategies have been used to control WSSV, each with some limitations. Conventional control strategies such as improvement of environmental conditions, stocking of pathogen-free post-larvae, and augmentation of disease resistance by oral immune-stimulants or probiotics are currently employed to control WSSV infection. Use of recombinant viral proteins as vaccines that induce a specific immune response and protection has been demonstrated to control WSSV. Other studies have shown successful vaccination of shrimp with DNA vaccines that have prolonged effects. The RNA interference (RNAi) mediated silencing of targeted viral mRNAs holds tremendous potential for controlling shrimp diseases. The silencing of viruses using RNAi has been experimentally demonstrated for WSSV in shrimp by injecting or feeding synthetic siRNA, long double-stranded RNA (dsRNA), and short/long-hairpin RNA (shRNA/lhRNA) prepared by in vitro transcription or expressed in bacteria. In addition to targeting viral proteins, protection of WSSV has also been achieved by dsRNA targeted against shrimp PmRab7, a protein important for viral entry into the host cells. Antisense constructs offered strong protection in WSSV challenged shrimp, P. monodon, with a corresponding decrease in viral load. Antisense constructs expressing VP24 and VP28 offered the best protection with a consistent reduction in WSSV copy number in both cell culture and in experimental shrimp. The advantage of using antisense constructs is their lack of toxicity and immunogenicity and their high specificity towards the desired target. The usage of edible pellet feed coated with dsRNA against WSSV has shown promising results. Overall, the present investigation clearly demonstrates that it is possible to induce strong protection in shrimp against WSSV infection using host promoter-driven antisense constructs in controlled laboratory-scale experiments. However, it is important to develop a simple and efficient delivery system for extending this study to the field level.
\end{abstract}

Keywords: shrimp diseases; P. monodon; RNA interference; disease resistance.

(C) 2020 by the authors. This article is an open-access article distributed under the terms and conditions of the Creative Commons Attribution (CC BY) license (https://creativecommons.org/licenses/by/4.0/). 


\section{Funding}

This research received no external funding.

\section{Acknowledgments}

This research has no acknowledgment.

\section{Conflicts of Interest}

The authors declare no conflict of interest. 